\title{
Activity of putative orexin neurons during cataplexy
}

\author{
Shi Zhou ${ }^{1,2}$, Akira Yamashita', Jingyang Su ${ }^{1,2}$, Yang Zhang ${ }^{1,3}$, Wuyang Wang ${ }^{3}$, Liying Hao ${ }^{2}$, \\ Akihiro Yamanaka ${ }^{4}$ and Tomoyuki Kuwaki ${ }^{*}$ (i)
}

\begin{abstract}
It is unclear why orexin-deficient animals, but not wild-type mice, show cataplexy. The current hypothesis predicts simultaneous excitation of cataplexy-inhibiting orexin neurons and cataplexy-inducing amygdala neurons. To test this hypothesis, we measured the activity of putative orexin neurons in orexin-knockout mice during cataplexy episodes using fiber photometry. We created two animal models of orexin-knockout mice with a GCaMP6 fluorescent indicator expressed in putative orexin neurons. We first prepared orexin-knockout mice crossed with transgenic mice carrying a tetracycline-controlled transactivator transgene under the control of the orexin promoter. TetO-GCaMP6 was then introduced into mice via an adeno-associated virus injection or natural crossing. The resulting two models showed restricted expression of GCaMP6 in the hypothalamus, where orexin neurons should be located, and showed excitation to an intruder stress that was similar to that observed in orexin-intact mice in our previous study. The activity of these putative orexin neurons increased immediately before the onset of cataplexy-like behavior but decreased (approximately $-20 \%$ of the baseline) during the cataplexy-like episode. We propose that the activity of orexin neurons during cataplexy is moderately inhibited by an unknown mechanism. The absence of cataplexy in wild-type mice may be explained by basal or residual activity-induced orexin release, and emotional stimulus-induced counter activation of orexin neurons may not be necessary. This study will serve as a basis for better treatment of cataplexy in narcolepsy patients.
\end{abstract}

Keywords: Cataplexy, Narcolepsy, Fiber photometry, Orexin knockout mice, Positive emotion

\section{Introduction}

Cataplexy is an emotionally triggered loss of muscle strength and postural collapse threatening daily life of narcolepsy patients. Cataplexy is a major symptom of narcolepsy, which is caused by abnormal loss of orexin (hypocretin)-producing neurons in humans [1], orexindeficiency in mice [2], and orexin receptor mutation in dogs [3].

It is unclear why the absence of orexin neuronal transmission results in cataplexy. The current hypothesis holds

\footnotetext{
*Correspondence: kuwaki@m3.kufm.kagoshima-u.ac.jp

1 Department of Physiology, Kagoshima University Graduate School of Medical and Dental Sciences, Kagoshima 890-8544, Japan

Full list of author information is available at the end of the article
}

that it involves simultaneous excitation of cataplexyinhibiting orexin neurons and cataplexy-inducing amygdala neurons [4-7]. An increase in orexin levels in the amygdala reduces cataplexy [5], indicating a cataplexyinhibiting role for orexin neurons. Furthermore, activation of orexin neurons results in the inhibition of the amygdala via the activation of serotonin neurons in the dorsal raphe, which receive orexinergic innervation $[6,8]$. Lesion or inactivation of the amygdala in orexin-deficient mice reduced cataplexy $[4,6]$, indicating a cataplexyinducing role for amygdala neurons. A microdialysis study showed orexin spillover with positive emotions in the amygdala of human patients with resistant epilepsy (but not narcolepsy) [9]. Although these observations fit the above hypothesis well, excitation of orexinergic 
neurons during cataplexy episodes in a time resolution of seconds has never been observed.

In addition to the awake-stabilizing role of orexin, orexin neuronal activity is closely linked to sympathetic autonomic outflow $[10,11]$. Therefore, changes in the heart rate during cataplexy may be a good indicator of the activity of orexin neurons. In this context, a decrease in heart rate during cataplexy has been reported in narcolepsy patients [12] and narcolepsy dogs [13], indicating the inhibition of orexin neuronal activity during cataplexy, which is in contrast to the abovementioned counterbalance activation hypothesis.

We recently succeeded in assessing real-time orexin neuronal activity along with electrocardiograms in freely behaving mice using a fiber photometry system [11]. Using this system, we reported that aversive stimulusevoked activation of orexin neurons preceded a heart rate increase. As an extension of the system, we hypothesized that it would be possible to assess putative orexin neuronal activity in orexin-knockout mice during cataplexy. Therefore, in this study, we measured putative orexin neuronal activity in two orexin-knockout mice models during cataplexy given that both activation and inhibition can be predicted from available circumstantial evidence.

\section{Results}

\section{Validation of the model animals}

We used six model 1 mice (Fig. 1A1: ORX ${ }^{-1-}$; ORXtTA mice injected with AAV-GCaMP6 and AAVmCherry) and four model 2 mice (Fig. 1A2: $\mathrm{ORX}^{-1-}$; ORX-tTA;TetO-GCaMP6). However, we excluded the data from three animals (two model 1 and one model 2) because of failure in adeno-associated virus (AAV) injection or optic fiber implantation was noted on histological examination. In valid cases, model 1 animals showed almost exclusive expression of GCaMP6 and mCherry in the hypothalamus, where orexin neurons should be located, and the area under the successfully implanted optic fiber (Fig. 1B1; double in GCaMP6 $=74.9 \pm 2.1 \%$, double in mCherry $=91.4 \pm 1.5 \%$, mean \pm SEM, $n=4)$. In model 2 animals, there were $87.3 \pm 7.3$ (mean \pm SEM, $\mathrm{n}=3$ ) GCaMP6-positive cells per side in a slice. This number was slightly larger than that in model 1 $(72.5 \pm 5.1, \mathrm{n}=4)$, but the difference was not statistically significant $(\mathrm{p}=0.176$, Student's $t$-test). We further confirmed that GCaMP6-positive cells in model 2 mice were putative orexin neurons, showing almost exclusive expression of GCaMP6 in orexin-immunoreactive cells, with more than $90 \%$ of these cells expressing GCaMP6 in $\mathrm{ORX}^{ \pm}$; ORX-tTA;TetO-GCaMP6 mouse (Fig. 1B2', $\mathrm{n}=1$ ). Thus, the fluorescence data described below were considered to originate from putative orexin neurons from a histological point of view.
To confirm that our data came from putative orexin neurons with physiological meanings, we recorded GCaMP6 and mCherry fluorescent signals together with ECG when the animals were confronted with intruder stress (Fig. 2). On exposure to intruder stress, GCaMP6 signals during the first $1 \mathrm{~min}$ increased significantly from $100.0 \pm 0.02 \%$ to $135.4 \pm 5.5 \% \quad(n=3, p=0.017$, two-way ANOVA followed by Sidak's multiple comparisons test) in model 1 animals and from $99.9 \pm 0.11 \%$ to $136.7 \pm 7.4 \%(\mathrm{n}=2, \mathrm{p}=0.027)$ in model 2 animals. There was no statistically significant difference between the models $\left(\mathrm{F}_{1,3}=0.0214, \mathrm{P}=0.893\right)$. In a similar manner, the heart rate increased from $471.2 \pm 3.9 \mathrm{bpm}$ to $539.6 \pm 5.4 \mathrm{bpm}(\mathrm{p}=0.002)$ in model 1 animals and from $461.2 \pm 15.5 \mathrm{bpm}$ to $544.5 \pm 4.0 \mathrm{bpm}(\mathrm{p}=0.002)$ in model 2 animals. There was no statistically significant difference between the models $\left(\mathrm{F}_{1,3}=0.075, \mathrm{p}=0.802\right)$. In contrast, the mCherry signal (measured only in model 1 animals) did not change $(100.1 \pm 0.07 \%$ vs. $100.0 \pm 0.14 \%$, $\mathrm{n}=3, \mathrm{P}=0.498$ ). Thus, the fluorescence sources in models 1 and 2 were similar. More importantly, these values were similar to those obtained in orexin-preserved mice $\left(\mathrm{ORX}^{+/+}\right.$; ORX-tTA mice injected with AAV-GCaMP6 and AAV-mCherry) in our previous study [11] ( $\triangle \mathrm{GCaMP}$ ca. $40 \%, \Delta$ mCherry ca. $0 \%)$. Thus, the fluorescence data shown below were considered to originate from putative orexin neurons from both histological and physiological points of view.

\section{GCaMP fluorescence and heart rate increases immediately} before and decreases during cataplexy-like behavior

Cataplexy-like behavior was observed $31.8 \pm 2.9$ times in model 1 animals $(n=4)$ and $27.9 \pm 3.0$ times in the model 2 animals $(n=3)$ during a 10-h observation period. There was no statistically significant difference between the models $(\mathrm{p}=0.405)$. Traces of heart rate and fluorescence were aligned so that the onset time of cataplexy-like behavior was equal and the averaged values of the time were calculated for all cataplexy-like behaviors in a single animal (Fig. 3). There was a significant increase in heart rate (from $567.7 \pm 5.3 \mathrm{bpm}$ to $623.9 \pm 5.3 \mathrm{bpm}, \mathrm{p}<0.0001$, $\mathrm{n}=25$ ) around the onset of cataplexy-like behavior and a significant decrease (to $512.0 \pm 4.7 \mathrm{bpm}, \mathrm{p}<0.0001$ ) during the cataplexy-like episode. In a similar manner, GCaMP6 fluorescence increased (from $100.0 \pm 0.1 \%$ to $113.2 \pm 1.2 \%, \mathrm{P}<0.0001, \mathrm{n}=25)$ almost immediately before the heart rate peak and then decreased (to $89.0 \pm 1.1 \%, \mathrm{p}<0.0001)$ during cataplexy-like behavior. The GCaMP6 fluorescence peak $(10.01 \pm 1.33 \mathrm{~s}$ before the onset of cataplexy-like behavior) significantly preceded the heart rate peak $(1.50 \pm 0.47 \mathrm{~s}, \mathrm{p}<0.0001$, $\mathrm{n}=25$, paired $\mathrm{t}$-test). In contrast, mCherry fluorescence did not show any change. There was no apparent 
A1

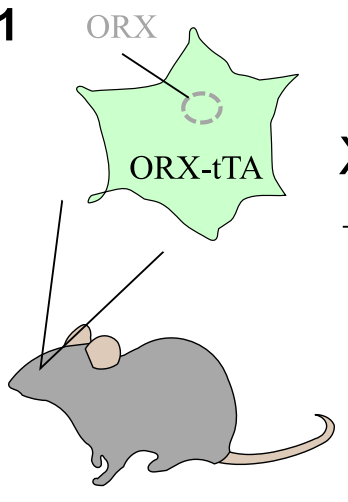

ORX---OORX-tTA
AAV dj-TetO(3G)-GCaMP6

AAV dj-TetO(3G)-mCherry

X

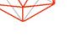

Human orexin
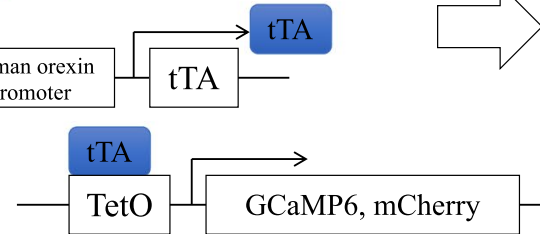

ORX-1-;ORX-tTA;

TetO-GCaMP6

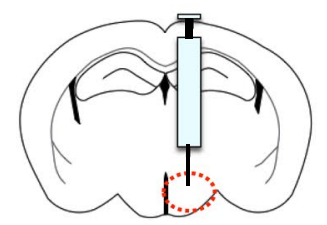

A2
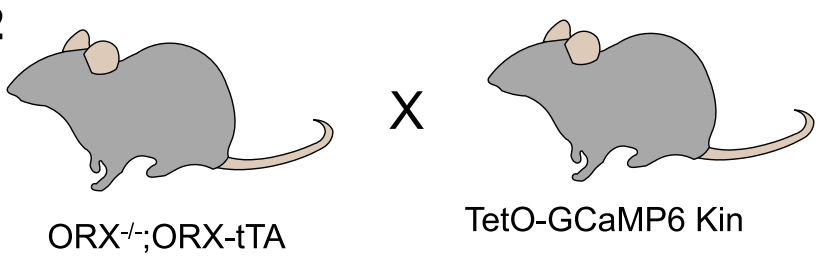

TetO-GCaMP6 Kin

B1 ${ }^{\mathrm{G}-\mathrm{CaMP} 6}$ \& mCherry in $\mathrm{ORX}^{-/-} ; \mathrm{ORX}-\mathrm{tTA}+\mathrm{AAV}$

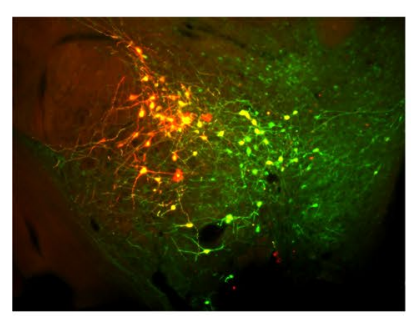

B2 G-CaMP6 \& ORX in ORX ${ }^{-/}$; ORX-tTA;TetO-GCaMP6

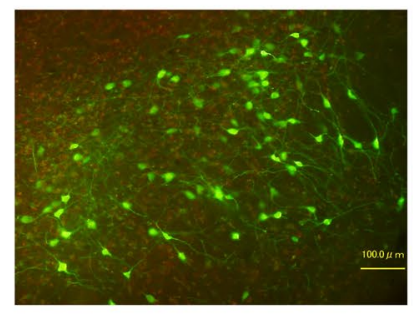

B2' G-CaMP6 \& ORX in ORX ${ }^{+/-}$;
ORX-tTA-TetO-GCaMP6
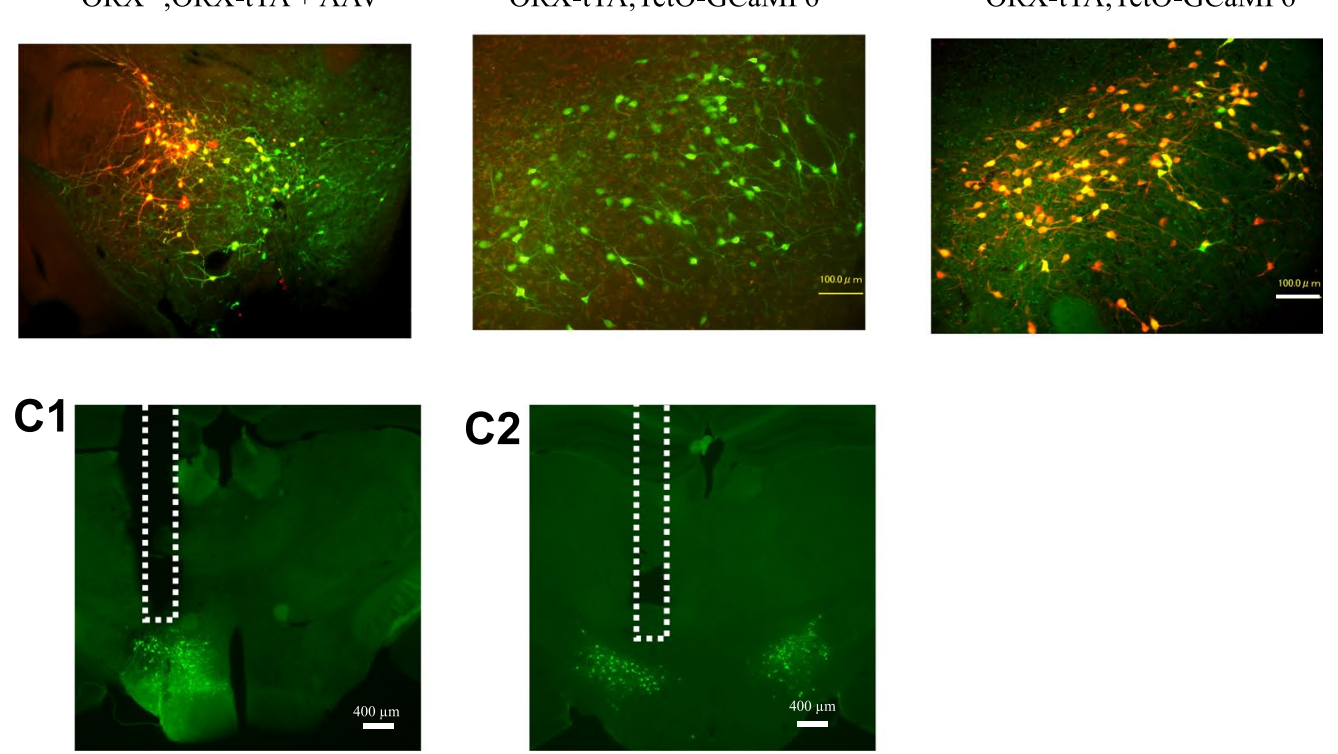

Fig. 1 Animal models for measuring putative orexin neuronal activity. A1 Schematic drawing showing specific expression of GCaMP6 and

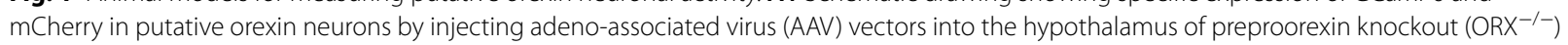
and orexin-tTA transgenic (ORX-tTA) double-mutant mice. We refer to this animal as "model 1" in this study. A2 Schematic drawing showing specific expression of GCaMP6 in putative orexin neurons by crossing double mutants of ORX ${ }^{-1-}$ and ORX-tTA with TetO-GCaMP knock-in mice. We call this animal "model 2" in this study. B1 Histological confirmation showing overlapping distribution of GCaMP6 (green) and mCherry (red) in the hypothalamus of a model 1 mouse. B2 Histological confirmation showing GCaMP6 (green) in the hypothalamus of a model 2 mouse. Note that no orexin-like immunoreactivity was found, as expected. B2' Histological confirmation showing overlapping distribution of GCaMP6 (green) and orexin-like immunoreactivity (red) in the hypothalamus of a mouse carrying the orexin-knockout heterozygous allele in model 2. C1 \& C2 Confirmation of fiber tracts after fluorescent recordings. The dashed line indicates the location of the inserted optic fibers. The left panel C1 shows a brain from a model 1 mouse and the right panel $\mathbf{C} 2$ shows a brain from a model 2 mouse 


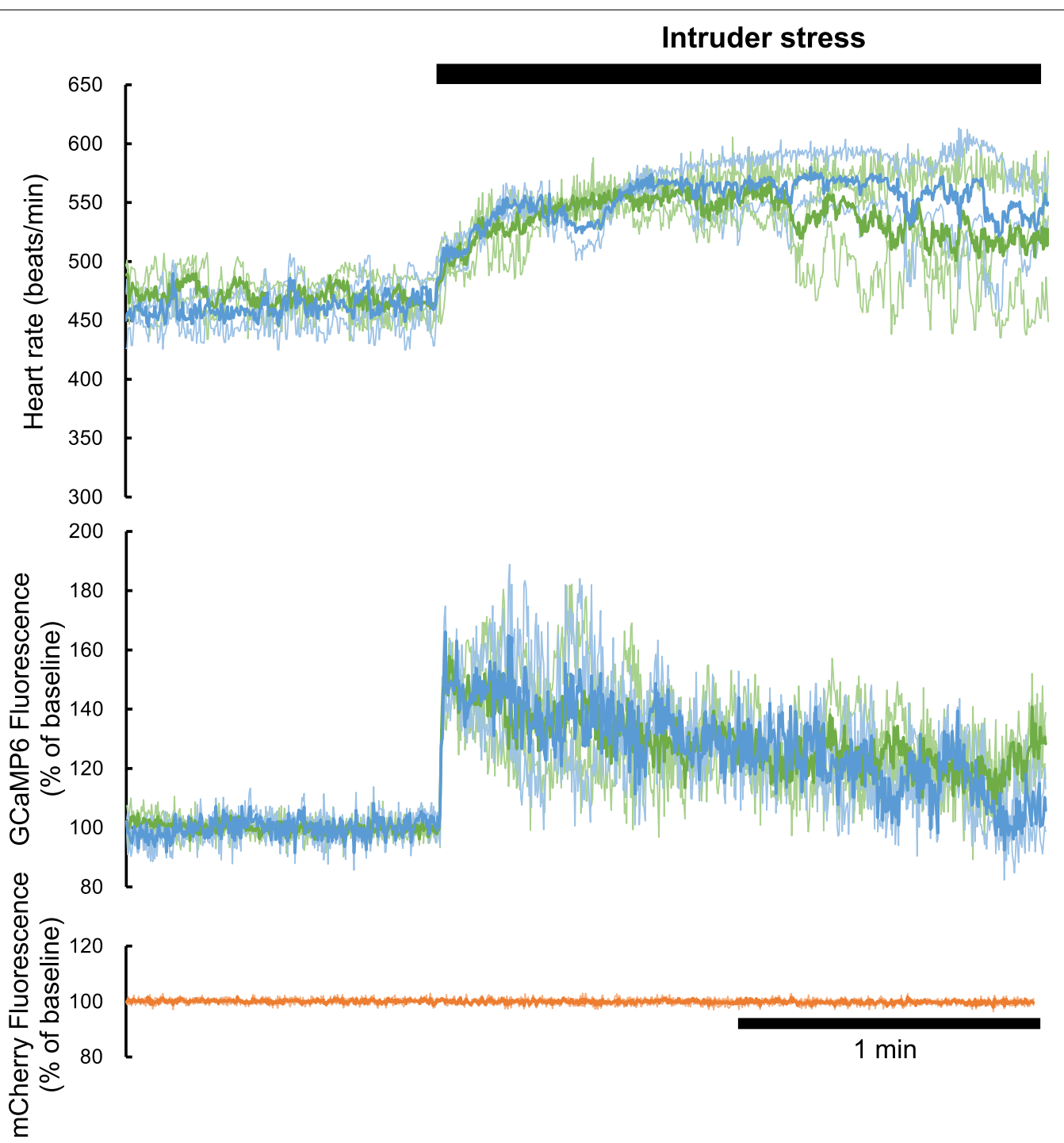

Fig. 2 Physiological confirmation showing activation of putative orexin neurons to intruder stress. The time course of the heart rate, GCaMP6 fluorescence, and mCherry fluorescence during intruder stress are shown from the top to the bottom. We used three model 1 animals, shown in green, and two model 2 animals, shown in blue. Thin lines indicate the data from individual animals, and thick lines indicate the averaged values. Note that the averaged responses in models 1 and 2 were almost similar for both heart rate and GCaMP fluorescence. mCherry data were only obtained in three model 1 animals and showed no change on exposure to the intruder

difference in the time course of changes in heart rate and GCaMP6 fluorescence among individual cataplexy-like episodes, except that they varied relatively widely during the latter part of the recordings, as shown in Fig. 3, presumably because the duration of cataplexy-like episodes varied from episode to episode $(27-209 \mathrm{~s}$ in the animal in Fig. 3).

Using the averaged data for all cataplexy episodes in one animal (see Fig. 3) as representative data for that animal, we constructed grouped data ( $\mathrm{n}=7$ animals) during cataplexy-like episodes (Fig. 4). The baseline value was defined as the average during a 30 -s period from
$60 \mathrm{~s}$ before to $30 \mathrm{~s}$ before the onset of cataplexy behavior. The maximum and minimum data were defined as the average of $5 \mathrm{~s}$ around the peak. Grouped data again showed a significant increase (Fig. 4, right panel) in heart rate around the onset of cataplexy-like behavior and a significant decrease during the cataplexy-like episode. GCaMP6 fluorescence increased almost immediately (ca. $10 \mathrm{~s}$ ) before the onset of cataplexy-like behavior and the maximum peak of the heart rate, and then decreased during cataplexy-like behavior. Cataplexy-like behavior lasted for $87.7 \pm 5.1 \mathrm{~s}$ (average of 7 animals), and GCaMP fluorescence returned to baseline $154.6 \pm 8.5 \mathrm{~s}$, about $70 \mathrm{~s}$ 


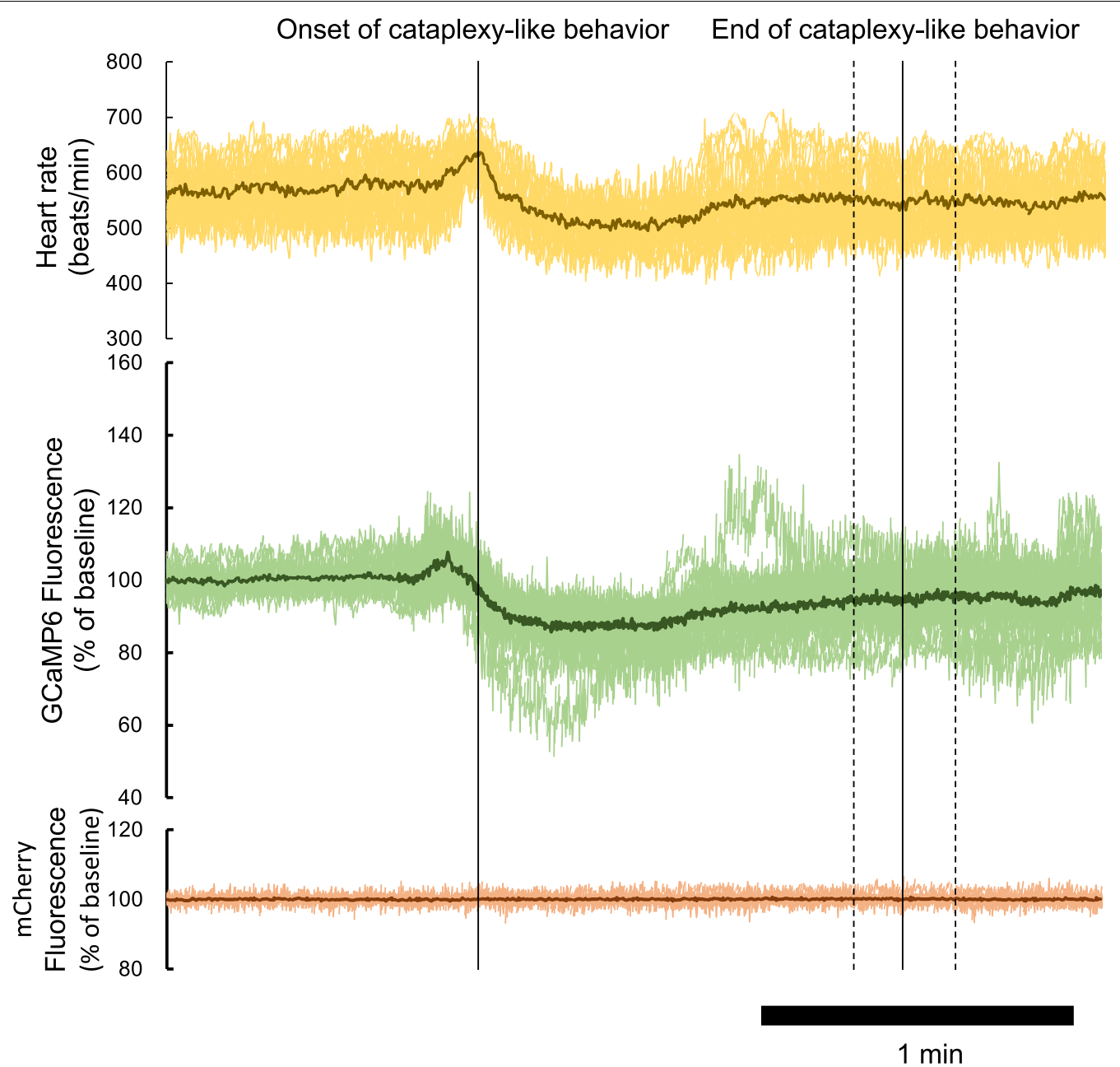

Fig. 3 Typical tracing of the heart rate, GCaMP fluorescence, and mCherry fluorescence in a model 1 animal. The time course of the heart rate, GCaMP6 fluorescence, and mCherry fluorescence during cataplexy-like behavior are shown from the top to the bottom. During the 10-h observation period, 25 episodes of cataplexy-like behaviors were observed in this animal. Each record was aligned so that the onset time of cataplexy-like behavior was equal and is shown as overlapping thin lines. The average value from the 25 episodes is shown as a thick line. The cataplexy-like episode lasted $79.8 \pm 9.7 \mathrm{~s}$ (mean \pm SEM, range 27-209 s, dashed lines indicate the standard error of the mean)

after the recovery from cataplexy. During cataplexy-like episodes, GCaMP6 fluorescence did not reach zero, indicating the existence of some residual activity in putative orexin neurons.

\section{Discussion}

In this study, we described the transient excitation of putative orexin neurons immediately before the onset of cataplexy-like behavior and successive inhibition during the episode. These changes were followed by changes in heart rate.

\section{Validation of the model animals}

We used two strategies to express GCaMP6 in putative orexin neurons in orexin-knockout mice: one involved the use of an AAV vector and the other involved natural crossing. The resulting mouse lines showed close resemblance to each other from histological (location in the lateral and dorsal hypothalamic area) and physiological (response to intruder stress) points of view, indicating reasonable validity of the models. Most importantly, the changes in fluorescence of GCaMP6 and mCherry were similar to those obtained in orexin-preserved mice $\left(\mathrm{ORX}^{+/+}\right.$; ORX-tTA mice injected with AAV-GCaMP6 and AAV-mCherry) in our previous study [11] ( $\triangle$ GCaMP approximately $40 \%, \Delta$ mCherry approximately $0 \%$ ), indicating that intruder stress activated both intact orexin neurons and putative orexin neurons in a similar manner.

Changes in heart rate in the current study (ca. $70 \mathrm{bpm}$ ) were relatively smaller than in those reported in a 

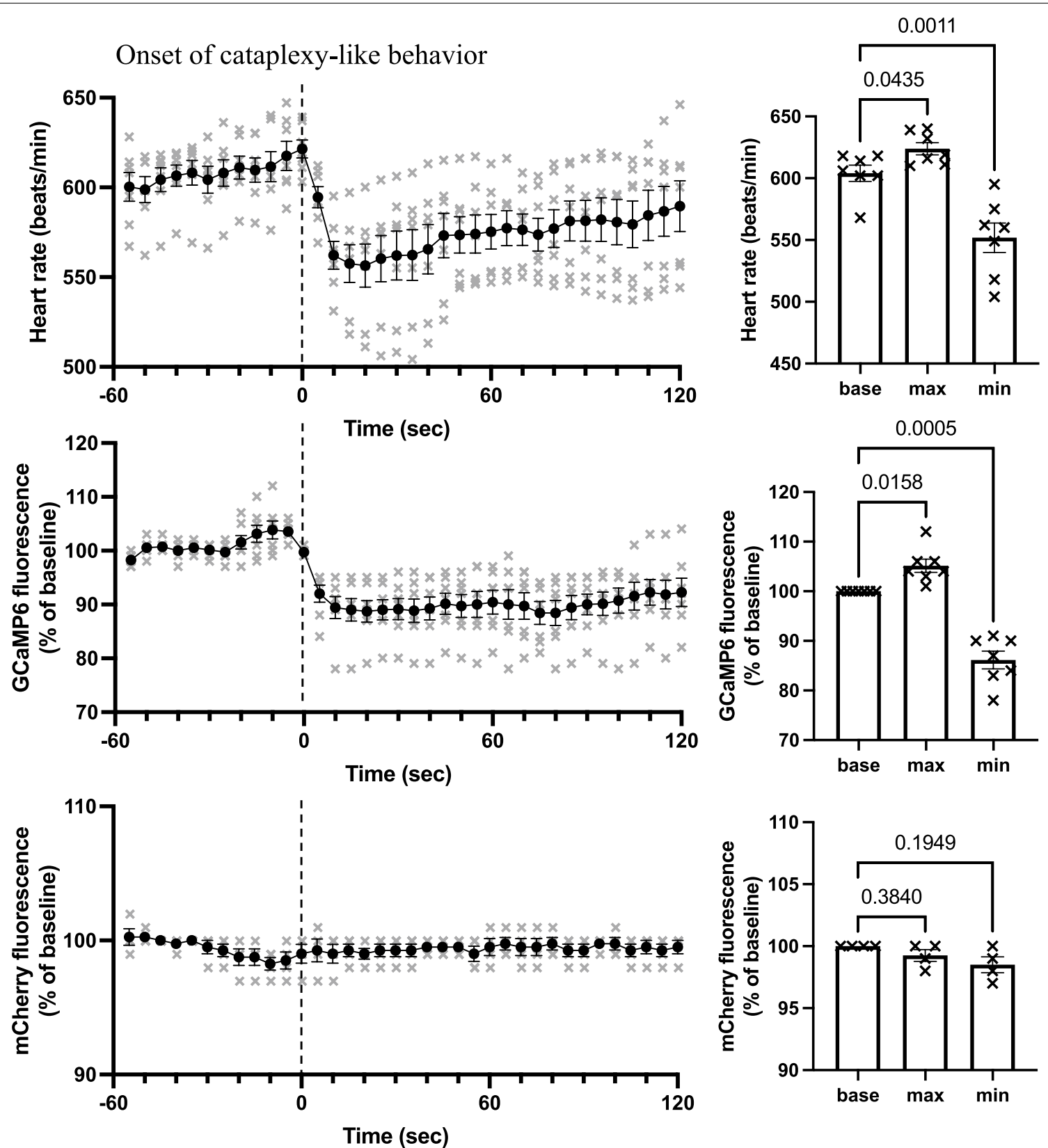

Fig. 4 Time-related changes in the heart rate, GCaMP fluorescence, and mCherry fluorescence grouped from seven animals. Left column: Time course of the heart rate, GCaMP6 fluorescence, and mCherry fluorescence during cataplexy-like behavior are shown from the top to the bottom. The averaged value in one animal for all of the cataplexy-like episodes (see thick line in Fig. 3) was resampled in 5-s bins and was averaged for seven animals. Circles denote mean values, and vertical lines denote the standard error of the mean. The individual values are indicated by crosses. Heart rate and GCaMP fluorescence data were obtained from four model 1 animals and three model 2 animals. mCherry data were obtained from four model 1 animals. Right column: Baseline value during the 30-60 s before, maximum value during the $0-30 \mathrm{~s}$ before, and minimum value during the $0-90 \mathrm{~s}$ after the onset of cataplexy were compared. There were significant differences in heart rate values $\left(F_{2,6}=37.26, p=0.0001\right)$ and $G C a M P 6$ values $\left(F_{2,6}=47.75, p=0.0001\right)$, but not in mCherry values $\left(F_{2,3}=4.765, p=0.1055\right)$. $P$ values in the figure were obtained using 1 -way analysis of variance, followed by Sidak's multiple comparison test

previous study [11] (ca. $150 \mathrm{bpm}$ ). This result indicated that orexin neurotransmitters from orexin neurons contributed substantially to the increase in heart rate [14] and that an orexin neuron-independent pathway, such as that observed in glutamatergic neurons in the dorsomedial hypothalamus [15], also helped increase the heart rate in response to intruder stress. 
During cataplexy-like behavior in this study, we found a marked decrease in heart rate (Fig. 4), which closely resembles that reported in narcolepsy patients [12] and narcolepsy dogs [13]. Therefore, we considered that the cataplexy episode could be well-estimated by the current method of behavioral observation, without assessment of the EEG. In dogs with narcolepsy [13] but not in human patients [12], an increase in heart rate at around the onset of a cataplexy attack has also been reported. Our mouse models closely resembled the dog cases. Differences in the deficit of orexin-signaling system (mice: deficiency of orexin peptide [2], dog: non-functional orexin receptor [3], human: ablation of orexin-synthesizing neurons [1]) may be related to species differences in the heart rate response because ablation of orexin-synthesizing neurons extinguishes not only orexin but also coexisting neurotransmitters/modulators expressed by the neurons $[16$, 17]. It may be interesting to note here that a functional magnetic resonance imaging study in narcolepsy patients showed marked reductions in hypothalamic activity during cataplexy attack [18], although the relevant neuronal identity was unclear.

\section{Inhibition of the putative orexin neurons during cataplexy}

Excitation of putative orexin neurons, as shown by an increase in GCaMP6 fluorescence (Figs. 3, 4), may fit the counterbalance hypothesis of simultaneous activation of cataplexy-promoting amygdala neurons and cataplexyinhibiting ORX neurons [4-7]. In this case, however, it seemed difficult to explain how short-lasting (ca. 15 s) excitation before the onset of cataplexy counteracts subsequent relatively long-lasting (30-200 s) cataplexy episodes. A short-lasting excitation may release ORX in the wild-type mice and amplify ORX neuronal activity through orexin-2 receptor expressed in the ORX neurons [19]. However, excitation of ORX neurons by orexins lasts for no more than $30 \mathrm{~s}$, at least in vitro [19]. We do not know whether such excitation, if any, might be enough to overcome succeeding inhibitory inputs to the orexin neurons. The second interpretation of the current results may be that the reduction of heart rate and GCaMP6 signals may indicate a transient slow-down of hyper arousal state with high sympathetic tones, because cataplexy often occurs in the middle of highly active behavior in ORX-deficient mice [2, 20, 21]. The lowered signals, especially the heart rate $(\sim 550 \mathrm{bpm}$, Fig. 4$)$, may show even higher level than those seen during the quiet wake period $(\sim 470 \mathrm{bpm}$, before intruder stress in Fig. 2); thus, these reductions may simply reflect the end of hyper activity of ORX knockout mice before a cataplexy episode. If this interpretation was true, then putative ORX neurons in ORX knockout mice seemed to not receive counter activation, at least during the cataplexy period. The third interpretation of the current results may be that cataplexy-inhibiting orexin neurons are subpopulations of ORX neurons, and even when these neurons are excited during cataplexy, inhibition of other subpopulations of ORX neurons mask such excitation. Since orexin neurons play multiple roles such as wakepromotion, food-seeking, motivation, and sympathetic activation [22, 23], some researchers have proposed the existence of subpopulations [24]. However, even if there are subpopulations of ORX neurons, the possible relationship between the activity of the subpopulation and cataplexy attack is highly speculative at present. The fourth and most simple explanation is that low activity of ORX neurons, if not zero, is sufficient to suppress cataplexy in wild-type mice. This explanation is supported by the observation that partial $(<90 \%)$ destruction of ORX neurons did not result in cataplexy [25]. Partial inhibition of putative ORX neuronal activity in this study indicated partial inhibition, but not excitation, of ORX neuronal activity in the wild-type mice in a situation where cataplexy would occur in the ORX knockout mice. Our notion is supported by evidence that partial blockade of orexin receptors by hypnotic drugs did not induce cataplexy [26] and that ectopic supplementation of ORX is effective in reducing cataplexy in ORX neuron-ablated mice [27].

\section{Limitations}

At this time, we do not know how and why orexin neurons receive inhibitory input during cataplexy. Orexin neurons express opioid receptors, and activation of the receptor inhibits orexin neuronal activity at least in vitro [28]. Positive emotions induce both cataplexy [21, 29] and opioid release [30]. Sympathetic activation, a role of orexin neurons [31], is not as strong during negative emotions as during positive emotions [32]. These factors may underlie the mechanism, but the biological meaning of this inhibition during cataplexy requires further study.

\section{Conclusions}

Here, we described the transient excitation of putative orexin neurons immediately before the onset of cataplexy-like behavior and their subsequent inhibition during the cataplexy-like episode. These results were not in good agreement with the counterbalance hypothesis. We propose a simpler hypothesis as follows. The absence of cataplexy in wild-type mice may be explained by basal or residual activity-induced orexin release, and emotional stimulus-induced counter activation of orexin neurons may not be necessary. This study will serve as a basis for better treatment of cataplexy in narcolepsy patients. 


\section{Materials and methods Ethics approval}

All experiments were conducted at Kagoshima University according to the guiding principles for the care and use of animals in the field of physiological sciences, published by the Physiological Society of Japan (2015), and were approved by the Experimental Animal Research Committee of Kagoshima University (MD17105 and MD20004).

\section{Animals}

We used two genetically engineered animal models of narcolepsy, which express the calcium indicator GCaMP6 in their putative orexin neurons. First, prepro-orexin-knockout mice [2] $\left(\mathrm{ORX}^{-/-}\right)$were crossed with transgenic mice carrying a tetracycline-controlled transactivator transgene (tTA) under the control of the orexin promoter [11] (ORX-tTA). The resulting $\mathrm{ORX}^{-1-}$; ORX-tTA mice were expected to express tTA, but not orexin, in their "orexin" neurons (Fig. 1A1). By injecting AAV-GCaMP6 (stereotaxic injection of $A A V$ section) into the hypothalamus of these mice, we obtained $\mathrm{ORX}^{-1-}$; ORX-GCaMP6 (model 1) mice. The second model was constructed by crossing $\mathrm{ORX}^{-1-}$; ORXtTA mice with TetO-GCaMP6 mice (Fig. 1A2). In TetO-GCaMP6 knock-in mice, the beta-actin gene was modified to convey a gene encoding tetracycline operator (TetO)-GCaMP6 [33] (B6; 129-Actb $<$ tm3.1(tetOGCaMP6)Kftnk > , obtained from RIKEN Bio Resource Research Center, RBRC09552). We used these two models to examine whether these two models, which are conceptually the same but technically different, would yield similar results.

All mice were housed in a room maintained at $22-24{ }^{\circ} \mathrm{C}$ with lights on at 19:00 and off at 7:00 for at least 2 weeks before experimentation began. We selected a reversed light/dark cycle so that the experimenters could observe mice in their active nocturnal phase of behavior during the daytime. We used male mice to avoid possible effects from an estrous cycle, as female hormonal cycles, at least in humans, may have some effect on sleep-related disorders, including narcolepsy with cataplexy [34]. We housed mice individually from the start of the reversed light/dark cycle to avoid any possible social rank effect on male behavior [35].

\section{Stereotaxic injection of AAV}

Under isoflurane anesthesia (2\%, inhalation) using a stereotaxic instrument (ST-7, Narishige, Tokyo, Japan), a viral mixture consisting of recombinant AAV-tetO(3G)G-CaMP6 (serotype: DJ; $600 \mathrm{nl} /$ injection, $3 \times 10^{12}$ copies/ $\mathrm{ml}$ ) and AAV-tetO(3G)-mCherry (serotype: DJ; $600 \mathrm{nl} /$ injection, $6 \times 10^{12}$ copies $/ \mathrm{ml}$ ) was stereotaxically injected into the left side of the hypothalamic perifornical area in $\mathrm{ORX}^{-/-}$;ORX-tTA mice (Fig. 1A1). All AAVs used in this study were produced by Yamanaka's Laboratory at Nagoya University, Japan [11]. The injection site was as follows: $1.5-\mathrm{mm}$ posterior from the bregma, $0.8-\mathrm{mm}$ lateral, and 5.0-mm ventral from the dura.

\section{In vivo recordings of neuronal activity using a fiber photometry system and cardiovascular parameters using a radio-telemetry system}

We used our previously reported method [11]. In brief, 2-3 weeks after viral injection (model 1 ) or 1 week before the measurement (model 2), mice were anesthetized with $2 \%$ isoflurane and were surgically implanted with a guide cannula (diameter: $600 \mu \mathrm{m}$, length of guide: $8 \mathrm{~mm}$, made by LUCIR, Tsukuba, Japan) to place the optical fiber immediately above the hypothalamus $(1.5 \mathrm{~mm}$ posterior to the bregma, $0.8 \mathrm{~mm}$ lateral, $4.0 \mathrm{~mm}$ ventral) to record orexin neuronal activity. Immediately after performing the guide cannula implantation, we performed additional surgery to implant a radio-telemetry transducer (TA11PA-C20, Data Sciences International, St. Paul, MN, USA) into the abdominal cavity of the mice to record electrocardiograms (lead II). During all surgeries, care was taken to maintain body temperature. After surgery, the mice were treated with penicillin, an analgesic, and buprenorphine. For recovery, mice were individually housed and monitored and had access to food and water ad libitum for at least 1 week.

A fiber photometry system (COME2-FTR/OPT, LUCIR, Tsukuba, Japan) was used to record the activity of orexin neurons in freely moving mice, as previously described [11]. During the measurement, a dummy fiber was removed and a 400- $\mu \mathrm{m}$ silica fiber (LUCIR) was inserted through the guide cannula into the brain. G-CaMP6 fluorescence and mCherry fluorescence were collected using the same silica fiber. The respective G-CaMP6 or mCherry fluorescence was guided to individual photomultipliers. The signal was digitized at $100 \mathrm{~Hz}$ using a data acquisition system (PowerLab16/35, ADInstruments, New South Wales, Australia) and was recorded using LabChart software version 8 (ADInstruments Inc., Bella Vista, NSW, Australia).

On the experimental day, mice were individually placed into a recording chamber in a soundproof box with a 12-h:12-h reversed light/dark cycle for $10 \mathrm{~h}$, from 8:00 (lights off at 7:00) to 18:00. The chamber was illuminated with a far infrared lamp (940 nm, SA2-IR, World Musen, Hong Kong), and a piece of chocolate was placed in the chamber at the start of observation to increase the episodes of cataplexy [21]. Mouse behavior was continuously recorded with a video camera (CBK21AF04, The Imaging Source Asia, Taipei, Taiwan) and was monitored 
on a personal computer located outside the soundproof box using the video capture function in LabChart. Electrocardiograms of mice were sampled through a radio-frequency receiver (RLA1020, Data Sciences International), digitally converted at $1000 \mathrm{~Hz}$, and transferred to a computer with PowerLab (ADInstruments). The heart rate was calculated using the cyclic variable function in LabChart. The video movie, G-CaMP6 and mCherry fluorescence signals, electrocardiogram, and calculated heart rate were stored on a personal computer. The fluorescence signal intensity at its nadir during the 10 -h observation period was defined as $0 \%$. At this moment, the mouse seemed to be in a sleeping/rest state, as judged through video recordings. To exclude individual differences in fluorescence signal intensity, the average value from -60 to $-30 \mathrm{~s}$ from the onset of cataplexy was defined as $100 \%$.

\section{Stress stimulation}

To test whether the putative orexin neuron in this study responded to an aversive stimulus, as did the orexin-producing neurons, we used an intruder stress test, as in our previous study [11]. This stressor was applied by placing an age-matched wild-type mouse (intruder mouse) in a small polypropylene cage into the experimental cage for $2 \mathrm{~min}$. The polypropylene cage was constructed such that the intruder and resident (experimental) mice were unable to contact each other physically, but visual, auditory, and olfactory communications were possible.

\section{Behavioral observation of cataplexy}

Cataplexy was determined according to the established criteria for mice [20], which are defined by several observable features. The first feature is an abrupt episode of nuchal atonia lasting at least $10 \mathrm{~s}$. Atonia was determined to occur when mice were in a prone position with their head and belly down in the bedding with their limbs and tail typically situated straight out from the trunk. This posture shows a clear contrast to a normal sleeping position in which mice are curled up and fold their limbs and tail underneath their trunk. Furthermore, in atonia, the mouse was immobile besides the movements associated with breathing during an episode. Finally, there had to be at least $40 \mathrm{~s}$ of active wakefulness (moving) preceding the atonia episode. The original criteria recommended recordings of EEG, but we did not adopt EEG to avoid heavy attachments to the head with the photometry fiber and EEG cables. Therefore, we refer to "cataplexylike behavior" instead of "cataplexy" in this manuscript.

\section{Immunohistochemistry}

Mice were deeply anesthetized with urethane $(2.0 \mathrm{~g} /$ $\mathrm{kg}$, i.p.) and were transcardially perfused with Ringer's solution (containing $\mathrm{CaCl}_{2}$ ), followed by $4 \%$ paraformaldehyde in $0.1 \mathrm{M}$ Tris $(\mathrm{pH} 7.4)+3 \mathrm{mM} \mathrm{CaCl}_{2}$. We added calcium to the ordinal washing and fixative solutions because in our preliminary experiment, we found that the fluorescence of GCaMP6 was better preserved with calcium supplementation. The brain was removed, post-fixed in 4\% paraformaldehyde + Ca solution at $4{ }^{\circ} \mathrm{C}$ overnight, and was subsequently immersed in phosphate buffered saline (PBS) at $4{ }^{\circ} \mathrm{C}$ for at least 2 days. A series of $40-\mu \mathrm{m}$ sections were obtained using a vibratome (SuperMicroSlicer Zero1; DOSAKA EM, Kyoto, Japan). For staining, coronal brain sections were immersed in blocking buffer (1\% normal horse serum and $0.3 \%$ Triton-X in PBS) and were then incubated with an anti-orexin A rabbit antibody (1:1000, 14346v, Peptide Institute, Osaka, Japan) at room temperature (about $20^{\circ} \mathrm{C}$ ) for $1 \mathrm{~h}$. The sections were washed with PBS and were incubated in a CF568-conjugated antirabbit IgG antibody (1:500, catalogue \#20098, Biotium, Heyward, CA, USA) for $2 \mathrm{~h}$ at room temperature. These brain sections were mounted on slides and were imaged using a fluorescence microscope (BZ-9000, Keyence, Osaka, Japan). For counting, we used one slice in which the optic fiber tract was most evident in the animal (Fig. 1C).

\section{Statistical analysis}

Statistical analyses were performed using GraphPad Prism (GraphPad Software, La Jolla, CA, USA). To compare the two groups of data, we used the Student's $t$-test. To compare three or more groups of means, we used oneway or two-way analysis of variance followed by Sidak's multiple comparison tests depending on the data structure. Data were reported as means and standard error of the mean. Statistical significance was set at $\mathrm{P}<0.05$.

\section{Acknowledgements \\ We thank members of the Department of Physiology for useful discussion and technical advice. We also acknowledge the Joint Research Laboratory and Laboratory of Animal Science at the Kagoshima University Graduate School of Medical and Dental Sciences for use of their facilities. \\ Authors' contributions \\ TK and A Yamashita designed the study; SZ, A Yamashita, JS, YZ, A Yamanaka, and TK conducted the study; A Yamashita, WW, LH, and TK analyzed the data; SZ, A Yamashita, and TK wrote the manuscript. All authors approved the final version of the manuscript. All authors read and approved the final manuscript. \\ Funding \\ This study was supported by JSPS KAKENHI (16H05130, 15K13112 to TK and $17 K 14936$ to A Yamashita), the Kodama Memorial Found for Medical Research (to A Yamashita), and CREST JST (JPMJCR1656 to A. Yamanaka).}

\section{Availability of data and materials}

Summary statistics are available in the article. The data that support the findings of this study are available from the corresponding author upon reasonable request. 


\section{Declarations}

Ethics approval and consent to participate

All experiments were conducted at Kagoshima University according to the guiding principles for the care and use of animals in the field of physiological sciences, published by the Physiological Society of Japan (2015), and were approved by the Experimental Animal Research Committee of Kagoshima University (MD17105 and MD20004).

\section{Consent for publication}

Not applicable.

\section{Competing interests}

The authors declare no competing interests.

\section{Author details}

'Department of Physiology, Kagoshima University Graduate School of Medical and Dental Sciences, Kagoshima 890-8544, Japan. ${ }^{2}$ Department of Pharmaceutical Toxicology, China Medical University, Shenyang 110122, Liaoning, China. ${ }^{3}$ Jiangsu Province Key Laboratory of Anesthesiology, Xuzhou Medical University, Xuzhou 221002, Jiangsu, China. ${ }^{4}$ Department of Neuroscience II, Research Institute of Environmental Medicine, Nagoya University, Nagoya 464-8601, Japan

Received: 10 January 2022 Accepted: 20 February 2022

Published online: 04 March 2022

\section{References}

1. Thannickal TC, et al. Reduced number of hypocretin neurons in human narcolepsy. Neuron. 2000;27:469-74.

2. Chemelli RM, et al. Narcolepsy in orexin knockout mice: molecular genetics of sleep regulation. Cell. 1999;98:437-51.

3. Lin $L$, et al. The sleep disorder canine narcolepsy is caused by a mutation in the hypocretin (orexin) receptor 2 gene. Cell. 1999;98:365-76.

4. Burgess $\mathrm{CR}$, et al. Amygdala lesions reduce cataplexy in orexin knock-out mice. J Neurosci. 2013;33:9734-42.

5. Liu M, et al. Orexin gene transfer into the amygdala suppresses both spontaneous and emotion-induced cataplexy in orexin-knockout mice. Eur J Neurosci. 2016;43:681-8.

6. Hasegawa $\mathrm{E}$, et al. Serotonin neurons in the dorsal raphe mediate the anticataplectic action of orexin neurons by reducing amygdala activity. Proc Natl Acad Sci USA. 2017:114:E3526-35.

7. Mahoney CE, et al. The neurobiological basis of narcolepsy. Nat Rev Neurosci. 2019:20:83-93.

8. Hasegawa E, et al. Orexin neurons suppress narcolepsy via 2 distinct efferent pathways. J Clin Invest. 2014;124:604-16.

9. Blouin AM, et al. Human hypocretin and melanin-concentrating hormone levels are linked to emotion and social interaction. Nat Com. 2013:4:1547.

10. Kuwaki T. Orexin links emotional stress to autonomic functions. Autonom Neurosci. 2011;161:20-7.

11. Yamashita A, et al. Aversive emotion rapidly activates orexin neurons and increases heart rate in freely moving mice. Mol Brain. 2021;14:104.

12. Donadio V, et al. Sympathetic and cardiovascular activity during cataplexy in narcolepsy. J Sleep Res. 2008;17:458-63.

13. Siegel $M$, et al. Heart rate and blood pressure changes associated with cataplexy in canine narcolepsy. Sleep. 1986;9:216-21.

14. Kayaba Y, et al. Attenuated defense response and low basal blood pressure in orexin knockout mice. Am J Physiol Regul Integr Comp Physiol. 2003;285:R581-93.

15. Kataoka N, et al. Psychological stress activates a dorsomedial hypothalamus-medullary raphe circuit driving brown adipose tissue thermogenesis and hyperthermia. Cell Metab. 2014;20:346-58.

16. Hara J, et al. Genetic ablation of orexin neurons in mice results in narcolepsy, hypophagia, and obesity. Neuron. 2001;30:345-54.

17. Zhang W, et al. Orexin neurons are indispensable for stress-induced thermogenesis in mice. J Physiol. 2010;588:4117-29.

18. Reiss AL, et al. Anomalous hypothalamic responses to humor in cataplexy. PLOS ONE. 2008:3:e2225.
19. Yamanaka A, et al. Orexin directly excites orexin neurons through orexin 2 receptor. J Neurosci. 2010;30:12642-52.

20. Scammell TE, et al. A consensus definition of cataplexy in mouse models of narcolepsy. Sleep. 2009;32:111-6.

21. Kuwaki T, Kanno K. Sexual excitation induces courtship ultrasonic vocalizations and cataplexy-like behavior in orexin neuron-ablated male mice. Comm Biol. 2021:4:165

22. Sakurai T. The role of orexin in motivated behaviors. Nat Rev Neurosci. 2014:15:719-31.

23. Kuwaki T. Orexin (hypocretin) participates in central autonomic regulation during fight-or-flight response. Peptides. 2021;139:170530.

24. Harris GC, Aston-Jones G. Arousal and reward: a dichotomy in orexin function. Trends Neurosci. 2006;29:571-7.

25. Tabuchi S, et al. Conditional ablation of orexin/hypocretin neurons: a new mouse model for the study of narcolepsy and orexin system function. J Neurosci. 2014:34:6495-509.

26. Tannenbaum PL, et al. Orexin receptor antagonist-induced sleep does not impair the ability to wake in response to emotionally salient acoustic stimuli in dogs. Front Behav Neurosci. 2014;8:182.

27. Mieda $M$, et al. Orexin peptides prevent cataplexy and improve wakefulness in an orexin neuron-ablated model of narcolepsy in mice. Proc Natl Acad Sci USA. 2004;101:4649-54.

28. Li Y, van den Pol AN. Mu -opioid receptor-mediated depression of the hypothalamic hypocretin/orexin arousal system. J Neurosci. 2008:28:2814-9.

29. Su J, et al. Involvement of the nucleus accumbens in chocolate-induced cataplexy. Sci Rep. 2020;10:4958.

30. Berridge KC, Kringelbach ML. Pleasure systems in the brain. Neuron. 2015;86:646-64.

31. Carrive P, Kuwaki T. Orexin and central modulation of cardiovascular and respiratory function. Curr Topics Behav Neurosci. 2016:33:157-96.

32. Ekman P, Levenson R, Friesen W. Autonomic nervous system activity distinguishes among emotions. Science. 1983;221:1208-10.

33. Ohkura $\mathrm{M}$, et al. Genetically encoded green fluorescent $\mathrm{Ca}^{2+}$ indicators with improved detectability for neuronal $\mathrm{ca}^{2+}$ signals. PLOS ONE. 2012; 7:e51286

34. Dijk D-J. Imaging and monitoring sleep and its disorders: local sleep, circadian rhythms and variability. J Sleep Res. 2012;21:485-6.

35. Shansky RM. Are hormones a "female problem" for animal research? Science. 2019:364:825-6.

\section{Publisher's Note}

Springer Nature remains neutral with regard to jurisdictional claims in published maps and institutional affiliations.

Ready to submit your research? Choose BMC and benefit from:

- fast, convenient online submission

- thorough peer review by experienced researchers in your field

- rapid publication on acceptance

- support for research data, including large and complex data types

- gold Open Access which fosters wider collaboration and increased citations

- maximum visibility for your research: over 100M website views per year

At BMC, research is always in progress.

Learn more biomedcentral.com/submissions 